\title{
VENEZUELA EN EL PROCESO DE INCORPORACIÓN AL MERCOSUR
}

\author{
Dani José Villalobos Soto ${ }^{[1]}$ \\ Enio Enrique Ortiz Valenzuela ${ }^{[2]}$
}

\section{Resumen}

Diversos han sido los esquemas de integración que han experimentado en sus prácticas los países de Latinoamérica, en su lucha por lograr una concentración de esfuerzos frente a las vicisitudes en aras de alcanzar intereses comunes para la región. Esta investigación enfoca al MERCOSUR como un proceso de integración que busca la conformación de un mercado común por medio de la eliminación de barreras arancelarias y para arancelarias. En ese sentido el presente estudio considera la participación de la República Bolivariana de Venezuela como posible miembro pleno del bloque, también se analiza el escenario asimétrico que caracteriza a Venezuela para su ingreso al MERCOSUR, donde se destacan las asimetrías de Venezuela para con los países miembros del bloque sureño, considerando de esta forma los potenciales beneficios y costos que tendrá para Venezuela la inclusión real al conglomerado de países que integran el MERCOSUR. Se ha basado en un trabajo de tipo documental, donde se realiza un análisis sobre la inclusión de Venezuela al esquema integracionista. Como conclusión es importante destacar que el objetivo del MERCOSUR se basa en crear una zona de libre comercio para beneficio de los países que integran América del Sur.

Palabras clave: Mercado Común, barreras arancelaria, barreras para arancelarias, balanza comercial, asimetrías.

\section{Summary}

Various scheme of integration have been experienced in practices of Latin American countries in their struggle to achieve a concentration of efforts against the vicissitudes in order to accomplish common interests for the region. This research focuses on MERCOSUR as an integration process that seeks the creation of a common market through the elimination of tariff wall and nontariff.

In this sense, the present study considers the participation of the Bolivarian Republic of Venezuela as a full member of the bloc; it also analyzes the asymmetric scenario that characterizes Venezuela for its entry into MERCOSUR, highlighting Venezuela's asymmetries with members of the southern bloc, thus considering the potential benefits and costs that it represent for Venezuela the real inclusion to the group of countries that are part of MERCOSUR.

\footnotetext{
${ }^{[1]}$ Profesor en Economía, dvillalobos@usb.ve

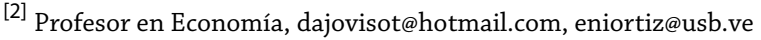


The present study is based on a documentary work, making an analysis on the inclusion of Venezuela to the integration scheme. In conclusion it is important to note that the goal of MERCOSUR is based on creating a free trade area for the benefit of the countries of South America.

Keywords: Common Market, tariff wall, nontariff wall, balance of trade, asymmetries.

\section{Introducción}

En Latinoamérica, a comienzos de la década de los sesenta, se dan los primeros pasos a la integración económica, cuyos objetivos se concentraban en fomentar mayores niveles de desarrollo y expansión de los países de la zona, a través de diversos mecanismos de intercambio de bienes y servicios entre sus miembros, con reducción de aranceles y mayor participación de sus sectores industriales estratégicos de penetrar los mercados de la región y su preparación previa para afrontar distintos escenarios internacionales. Parte de estas necesidades, sumando las sugerencias hechas por organismos de cooperación regional como la Comisión Económica para América Latina (CEPAL), mediante estudios propuestos para la sustitución de importaciones en la región, generaron importantes avances para dar origen a la Asociación Latinoamericana de Libre Comercio (ALALC), agrupación para la integración regional que buscaba el compromiso de los países socios de conformar una zona de libre comercio en un plazo máximo de 12 años desde su creación, el cual no pudo ser materializado en el tiempo previsto, debido a los diferentes niveles económicos de los países conformantes y posteriormente es reemplazado por la Asociación Latinoamericana de Integración (ALADI) en 1980, cuyo objetivo fundamental es ampliar y dinamizar los mecanismos de intercambio comercial establecidos por su antecesor, mediante la conformación de acuerdos más flexibles de carácter bilateral y multilateral entre sus socios, como por ejemplo los Acuerdos de Alcance Parcial y Acuerdos de Complementación Económica (ACE). No obstante, a partir de estos se generaron nuevas iniciativas de integración con mayor profundidad de compromisos de unificación de políticas comerciales y arancelarias y buscar un carácter más institucional a la integración económica Sudamericana, tales son los casos de: La Comunidad Andina (CAN) y El Mercado Común del Sur (MERCOSUR), además de una serie de Acuerdos Comerciales firmados entre los 12 países sudamericanos que actualmente son parte de la ALADI.

La adhesión de la República Bolivariana de Venezuela al MERCOSUR es un tema de actualidad, que ha dado mucho de que hablar debido a su complejidad del mismo y el exhaustivo análisis que se requiere para tomar la decisión de ingresar a un proceso de integración debido a los múltiples cambios que ello implica, así como también las reformas de las políticas comerciales las cuales son de gran importancia para la economía del país. 


\section{CIENCIAS SOCIALES}

Es en función a lo antes mencionado se refiere el presente artículo, las cuales tiene como objetivo explicar las obligaciones y las condiciones con que Venezuela ingresaría al MERCOSUR, así como también describir las asimetrías de carácter estructural y político que existen entre la República Bolivariana de Venezuela y los países miembros del MERCOSUR, y analizar la balanza comercia de Venezuela con el MERCOSUR. Para alcanzar los objetivos planteados el tipo de investigación es de tipo Documental, así pues, en base a esto y apoyo en trabajos anteriores, se pudo ampliar los conocimientos, estudiándose el problema y los objetivos propuestos.

\section{Desarrollo}

\section{Consideraciones generales}

Con el MERCOSUR se apertura un proceso de integración que se ha profundizado al pasar de los años a través de diferentes acuerdos y se está extendiendo con la figura de adhesión de Venezuela y la participación de otros países que no son Estados Partes del bloque económico, por tal motivo, se hará referencia a la evolución de los procesos de integración detallando los diferentes aspectos de los mismos, desde sus orígenes hasta el presente.

El fin de la Segunda Guerra Mundial y el deterioro que sufrieron las relaciones económicas internacionales a lo largo del período entre guerras, causó que todos los países aliados, con EEUU al frente, asumieran la responsabilidad de recomponer los elementos básicos de la economía mundial en el ámbito comercial, monetario y financiero. Por tal motivo, se crearon en la misma fecha, el Fondo Monetario Internacional (FMI) y el Banco Internacional de Reconstrucción y Desarrollo (BIRD) el 27 de diciembre de 1945 para regular las cuestiones monetarias y la movilización de capital a largo plazo. (M. Heredero, 2001)

Luego de este proceso se intentó crear la Organización Internacional de Comercio (OIC), la cual no fue posible por las crecientes discrepancias y distintos puntos de vista sobre facilitación del comercio existente entre Estados Unidos y las principales potencias de Europa Occidental. Al respecto M. Heredero (2001), señala: "aunque su creación fue realmente acordada en la Conferencia de la Habana, nunca llegó a ser ratificada por los países, especialmente por Estados Unidos; una paradoja, si se tiene en cuenta que fue el principal promotor de la idea." (p. 13).

Sin embargo, mientras seguían los trabajos preparatorios de la Carta de la OIC, los principales países llevaron a cabo negociaciones arancelarias para reducir aranceles y preferencias. A los efectos de estas negociaciones, dieron lugar en el año 1947 al Acuerdo General sobre Aranceles Aduaneros y Comercio mejor conocido por sus siglas en inglés como el GATT. Dicho acuerdo entró en vigor el 1 de enero de 1948. 
Para 1986 los compromisos se limitaban a la disminución de los derechos arancelarios a las importaciones y otros instrumentos de Política Comercial, cuyo propósito era la liberación del comercio internacional.

Dada la Ronda Uruguay del GATT, se introdujo a las negociaciones la idea de llegar a compromisos sobre una política de desregulación de los intercambios en el Sector Agrícola y de Servicios de los derechos de Propiedad Intelectual.

Para el año de 1994, dada la firma del Acuerdo de Marrakech, luego de ocho años de negociación en la Ronda Uruguay, se constituye la Organización Mundial del Comercio cuya operatividad se da inicio para el año 1995.

En la década de los 90 se crea el Mercado Común del Sur (MERCOSUR), mediante la firma del Tratado de Asunción el 26 de marzo de 1991 entre los países de Argentina, Brasil, Paraguay y Uruguay. Vásquez (2002) considera que:

...este Bloque económico fue creado con objetivos ambiciosos de los Estados Partes para lograr alcanzar la integración económica, y por ende la satisfacción de necesidades mutuas y competitivas...dichos países se constituyeron, no sólo para formar una Zona de Libre Comercio entre ellos, sino también una unión aduanera, con deseo de convertirse en un Mercado Común y constituirse en una de las formas más acabadas de integración... [Vásquez, C, 2002, p. 7](1).

De acuerdo a la cita anterior, dicho bloque fue constituido con la finalidad de ampliar las dimensiones de los mercados nacionales y con ello, acceder a los beneficios inherentes a la creación de un espacio económico ampliado.

Sin embargo, las diferencias entre los países socios del MERCOSUR son muy marcadas en varios aspectos, como el tamaño geográfico, la población y la actividad económica, lo cual son aspectos relevantes en todos los procesos de integración.

\section{Las obligaciones adquiridas y las condiciones con que Venezuela ingresaría al MERCOSUR}

Las exigencias del MERCOSUR para aprobar el ingreso de nuevos miembros, están descritas en el Tratado de Asunción, firmado en la Ciudad de Asunción, Paraguay. Este Tratado fue suscrito por la República Argentina, República Federativa del Brasil, República del Paraguay y República Oriental del Uruguay.

Dichas naciones establecen la ampliación de sus mercados nacionales, a través de la integración, constituyendo procesos para el desarrollo económico, alcanzando este beneficio por medio de los recursos disponibles y la coordinación de políticas 


\section{CIENCIAS SOCIALES}

económicas, comerciales y la complementación de los diferentes sectores de las economías, elevando la consolidación de grandes espacios para lograr una óptima consolidación del proceso integratorio de sus países componentes. Por lo cual, el acta constitutiva del MERCOSUR afirma que a través de tal proceso se alcanza la respuesta adecuada para tal fin.

En virtud de lo expuesto, el MERCOSUR promueve el desarrollo en diversos ámbitos y deja abierta la posibilidad para que nuevos socios puedan adherirse a sus filas.

El MERCOSUR plantea la posibilidad de adhesión de otros países, los cuales deben ser miembros de la ALADI. Si estos países solicitan el ingreso, los miembros del MERCOSUR, podrán analizar el caso, siempre y cuando los interesados no formen parte de otros acuerdos de integración sub-regional o de asociaciones extra-regionales. Considerando que la respuesta o aprobación debe ser de manera unánime por parte de: Brasil, Argentina, Uruguay y Paraguay.

Luego de haberse aprobado el proyecto de adhesión de Venezuela al MERCOSUR por los cuatro países fundadores en junio del año 2006, Venezuela se adhiere a dicho bloque mediante la firma del Protocolo de Adhesión

Según lo señalado en dicho protocolo, el estado venezolano desarrollará su integración en el MERCOSUR conforme a las obligaciones emanadas de este documento, persiguiendo los principios de gradualidad, flexibilidad y equilibrio considerando las asimetrías entre Venezuela y los países que integran el MERCOSUR, así como los principios de seguridad alimentaria, medios de subsistencia y desarrollo integral.

Hecha la consideración anterior, Venezuela se adhiere al MERCOSUR mediante este Protocolo. La República Bolivariana de Venezuela asume la normativa establecida en el Tratado de Asunción, Protocolo de Ouro Preto y el Protocolo de Olivos para la Solución de Controversias del MERCOSUR, herramientas que le permitirán a Venezuela poseer un mayor grado de participación en esta integración, ayudando a la ampliación del volumen comercial de productos venezolanos por productos de los países del MERCOSUR. De igual manera se establece en este artículo, que los Estados Partes del MERCOSUR se comprometen a realizar las modificaciones requeridas para que se pueda aplicar de manera correcta dicho Protocolo de Adhesión.

Por otra parte, la República Bolivariana de Venezuela debe regirse por las normativas actuales del MERCOSUR. La República Bolivariana de Venezuela deberá adoptar las normas actuales de MERCOSUR no de manera instantánea, sino de forma gradual, es decir, progresivamente pero nunca excediendo 4 años contados a partir de la aprobación y entrada en vigencia de dicho Protocolo. El cronograma en el que se estipule la adopción de la normativa será determinado por el Grupo de Trabajo del MERCOSUR. 
En este mismo orden de ideas, Venezuela debe adoptar la nomenclatura común del MERCOSUR y adaptar la misma al arancel y legislación aduanera nacional.

Se expresa que el Grupo de Trabajo determinará el cronograma en el cual Venezuela adoptará Nomenclatura Común del MERCOSUR (NCM) el cual es un código común del comercio internacional entre los Estados Partes, así como también la adopción del Arancel Externo Común (AEC), el cual es el sistema en donde se clasifican e identifican todo el universo de mercancías a nivel arancelario. Cada una de estas mercancías identificadas tiene adjudicado una tarifa Ad-Valorem, expresada en porcentaje, llamado Arancel que es el impuesto que pagará cada mercancía al ingresar al Mercosur por cualquiera de sus Estados Partes. Venezuela debe adoptar este instrumento a más tardar cuatro años después de la entrada en vigencia del protocolo de adhesión, proceso en cual Venezuela asume la normativa técnica arancelaria a emplear a todas las mercancías.

De igual manera, se establecen los plazos máximos en que los países deben alcanzar el libre comercio. Los países integrantes de este bloque económico reconociendo las asimetrías existentes entre ellos, se comprometen a alcanzar el libre comercio en diferentes períodos, los países de menor desarrollo económico relativo están favorecidos en cuanto al programa de liberación comercial. Estos plazos excluyen a los productos sensibles los cuales el plazo podrá prolongarse hasta el 1 de enero de 2014, fecha en que quedan sin efecto las normas y disposiciones previstas en el Acuerdo de Complementación Económica $\mathrm{N}^{\circ} 59$, entendiéndose como productos sensibles aquellos que por su impacto en la economía de un país no pueden verse beneficiados con el aceleramiento de la liberalización comercial establecidas en el Protocolo.

Ahora bien, los compromisos adquiridos por Venezuela al adherirse al Tratado de Asunción los estipula el artículo No. 1 del mismo.

Según el artículo No. 1, es evidente que Venezuela se verá obligada a reformar su actual Política Comercial para poder adaptarse y cumplir con las exigencias establecidas en el Tratado de Asunción por los países miembros del bloque y de esta manera ajustarse a la dinámica comercial dentro del MERCOSUR, la cual es común para todos sus miembros. Por esta razón, las decisiones respecto a la Política Comercial ya no se podrán tomar de manera unilateral, sino en conjunto.

Por otro lado el artículo No. 4 regula las relaciones comerciales de los miembros con terceros países.

Según este artículo, los Estados Partes coordinarán políticas comunes con la finalidad de evitar importaciones que ingresen bajo la aplicación de prácticas desleales como los subsidios, los cuales se refieren a cualquier contribución de carácter financiero, ayuda, prima, o premio dado por un gobierno o un organismo ya sea público o mixto, 
existente en un país extranjero, por medio de los cuales se le dé una ventaja a ciertas empresas o ramas de la producción, adicional a las que ya existen para otras empresas o ramas de la producción de comercio, las cuales afecten de una manera u otra los productos de los países socios en los diferentes mercados del bloque; o el dumping los cuales se refieren a la introducción de bienes o productos para su comercialización en el territorio nacional a un precio que se encuentra muy por debajo de su valor normal.

El artículo No. 8 establece algunas obligaciones que deben cumplir los países miembros a fin de preservar los compromisos adquiridos. (Tratado de Asunción). Según este artículo, los países miembros del MERCOSUR deben ser cuidadosos a la hora de celebrar cualquier otro acuerdo con restantes países miembros de la ALADI ya que deben evitar en lo posible que dicha celebración pueda afectar de una manera u otra los objetivos del Mercado Común; de igual manera cada vez que un país miembro del MERCOSUR negocie esquemas que contemplen la desgravación arancelaria lo cual es simplemente la reducción o disminución del porcentaje de los aranceles de importación o exportación, con la finalidad de formar zonas de libre comercio con otros países de la ALADI, debe consultar con los demás países miembros del MERCOSUR. Así mismo, se puede observar también en el artículo No. 8, como se aplica el principio de la nación más favorecida al estipular que cualquier país miembro debe extender automáticamente a los demás Estados Partes, cualquier ventaja que le conceda a un producto originario de o destinado a terceros países que no sean miembros de la ALADI.

Finalmente, el artículo No. 11 del Tratado de Asunción, establece las acciones a tomar por parte de los países miembros a fin de asegurar el cumplimiento del cronograma de desgravación. Se observa claramente, que una vez que los países forman parte del MERCOSUR se ven obligados a coordinar políticas macroeconómicas y sectoriales en conjunto para la constitución del Mercado Común empezando con los relacionados con los flujos de comercio y los sectores productivos.

\section{Descripción de las asimetrías de carácter estructural y político que existen entre Venezuela y los países miembros del MERCOSUR}

Venezuela, como cualquier otro país del mundo posee un nivel de desarrollo económico, tamaño geográfico, dotación de factores, ingreso per cápita, desarrollo de infraestructura, localización geográfica y políticas públicas, muy distintas a muchos países del mundo. En este caso se abordan las asimetrías que existen en Venezuela en comparación con los países miembros del MERCOSUR.

Así pues, dichas asimetrías condicionan la capacidad que tiene un país de poder aprovechar todos los beneficios que engloba un proceso de integración. Al respecto M. Terra, (2008) señala: 
La literatura sobre integración económica pone en evidencia que los beneficios de un proceso de integración son ambiguos y pueden distribuirse en forma desigual entre los socios. Para asegurar la viabilidad política de un proceso de integración se requiere que el mismo contribuya, o al menos no sea un obstáculo, para el crecimiento económico de sus socios. Por lo tanto, si las asimetrías entre los socios se convierten en un obstáculo para que los países puedan apropiarse de los beneficios del proceso, existiría cierto espacio para implementar políticas que atiendan este problema. [Terra, M., 2008, p. 192](2).

Como se ha dicho en capítulos anteriores, la economía distingue entre dos tipos de asimetrías, las estructurales y políticas. Según M. Terra, (2008) ambas son importantes. Las primeras, por razones obvias: se trata de un proceso de integración entre países que presentan grandes diferencias. Las segundas son consecuencias de la debilidad de la región en la correcta coordinación de políticas macroeconómicas y políticas de competencia, así como la escasez de consistencias al interior de los propios países en lo que se refiere a la aplicación de incentivos.

En este mismo sentido, como lo señalan Masi \& Alvarez (2005), Todo proceso de integración regional tiene como lugar de partida un conjunto de desigualdades de desarrollo y de competitividad entre los países miembros que comienzan dicho proceso. Y como los costos y los beneficios del proceso de integración de los mercados no siempre se distribuyen en iguales proporciones a través de los Estados Partes, incluso dentro de los propios Estados las desigualdades pueden perdurar durante el tiempo, e inclusive incrementarse. Por consiguiente, la profundización de las asimetrías preexistentes puede hacer política o económicamente insostenible el proyecto de integración.

A continuación, se presentan algunos indicadores Estructurales de Asimetrías entre los países miembros del MERCOSUR y Venezuela. (ver Cuadro No. 1)

Cuadro No. 1. Algunos indicadores Estructurales de Asimetrías entre el MERCOSUR y Venezuela, 2005

\begin{tabular}{|c|c|c|c|c|c|c|c|}
\hline & \multicolumn{6}{|c|}{ Participación en el total de la Comunidad Sudamericana de Naciones } \\
(Porcentaje) & \multicolumn{2}{c|}{$\begin{array}{c}\text { Desigualdades en } \\
\text { Producto Interno Bruto } \\
\text { por habitante (en miles } \\
\text { de dólares) }\end{array}$} \\
\hline & $\begin{array}{c}\text { Territorio } \\
\mathbf{( 1 7 . 7} \text { millo- } \\
\text { nes de km2) }\end{array}$ & $\begin{array}{c}\text { Población } \\
\text { (376.5 } \\
\text { millones de } \\
\text { habitantes) }\end{array}$ & $\begin{array}{c}\text { PIB (1.229 mil } \\
\text { millones de } \\
\text { dólares }\end{array}$ & $\begin{array}{c}\text { Comercio } \\
\text { Intrarregion } \\
\text { al (54.3 } \\
\text { millones de } \\
\text { dólares) }\end{array}$ & $\begin{array}{c}\text { Coeficiente } \\
\text { comercio } \\
\text { intra-CSN c/ } \\
\text { país }\end{array}$ & $\begin{array}{c}\text { En Moneda } \\
\text { Corriente }\end{array}$ & $\begin{array}{c}\text { En dólares de polaridad } \\
\text { de Compra (PPC) }\end{array}$ \\
\hline MERCoSUR & $\mathbf{6 7 . 0}$ & $\mathbf{6 2 . 6}$ & $\mathbf{6 5 . 3}$ & $\mathbf{6 6 . 4 8}$ & $\mathbf{2 2 . 1}$ & $\mathbf{3 4 0 2}$ & $\mathbf{9 2 1 4}$ \\
\hline Argentina & 15.6 & 10.2 & 12.9 & 25.90 & 35.1 & 4119 & 13830 \\
\hline Brasil & 48.0 & 49.8 & 50.6 & 37.68 & 17.3 & 3316 & 8405 \\
\hline Paraguay & 2.3 & 1.7 & 0.6 & 1.13 & 36.4 & 1197 & 4560 \\
\hline
\end{tabular}




\begin{tabular}{|c|c|c|c|c|c|c|c|}
\hline & \multicolumn{6}{|c|}{ Participación en el total de la Comunidad Sudamericana de Naciones } \\
(Porcentaje) & \multicolumn{2}{c|}{$\begin{array}{c}\text { Desigualdades en } \\
\text { Producto Interno Bruto } \\
\text { por habitante (en miles } \\
\text { de dólares) }\end{array}$} \\
\hline & $\begin{array}{c}\text { Territorio } \\
\text { (17.7 millo- } \\
\text { nes de km2) }\end{array}$ & $\begin{array}{c}\text { Población } \\
\text { (376.5 } \\
\text { millones de } \\
\text { habitantes) }\end{array}$ & $\begin{array}{c}\text { PIB (1.229 mil } \\
\text { millones de } \\
\text { dólares }\end{array}$ & $\begin{array}{c}\text { Comercio } \\
\text { Intrarregion } \\
\text { al (54.3 } \\
\text { millones de } \\
\text { dólares) }\end{array}$ & $\begin{array}{c}\text { Coeficiente } \\
\text { comercio } \\
\text { intra-CSN c/ } \\
\text { país }\end{array}$ & $\begin{array}{c}\text { En Moneda } \\
\text { Corriente }\end{array}$ & $\begin{array}{c}\text { En dólares de polaridad } \\
\text { de Compra (PPC) }\end{array}$ \\
\hline MERCOSUR & $\mathbf{6 7 . 0}$ & $\mathbf{6 2 . 6}$ & $\mathbf{6 5 . 3}$ & $\mathbf{6 6 . 4 8}$ & $\mathbf{2 2 . 1}$ & $\mathbf{3 4 0 2}$ & $\mathbf{9 2 1 4}$ \\
\hline Uruguay & 1.0 & 0.9 & 1.1 & 1.77 & 28.3 & 4024 & 9929 \\
\hline Venezuela & 5.1 & 7.1 & 9.6 & 4.87 & 4.8 & 4419 & 6152 \\
\hline
\end{tabular}

Fuente: Comisión Económica para América Latina y el Caribe (CEPAL) (2006).

Como se puede observar, las asimetrías que existen entre los países miembros del MERCOSUR y Venezuela en términos de territorio, población y PIB, Brasil y Argentina representan más del 90\% del total del bloque. Mientras que en el otro extremo se encuentran los dos países más pequeños (Paraguay y Uruguay) que no acumulan en conjunto ni el 4\% del PIB total. Por su parte Venezuela ocuparía el término medio entre los países más grandes y los más pequeños. Brasil y Argentina son los dos países gigantes en este bloque, presentando unas grandes diferencias asimétricas con Uruguay, Paraguay y Venezuela respecto al territorio, población, PIB y comercio interregional.

Sin embargo, se puede tener una visión más acertada de las asimetrías mencionadas anteriormente analizando el lugar que tienen los países en el ranking mundial que corresponden al Índice de Desarrollo Humano (IDH) elaborado por el Programa de Naciones Unidas para el Desarrollo. El IDH representa una aproximación más real al desarrollo debido a que incorpora tres dimensiones a considerar: expectativa de vida, nivel educativo y PIB por habitante. Aquí se observa que Argentina, el mejor posicionado de los países del MERCOSUR, ocupa el lugar 38, seguido de Uruguay, Brasil, luego Venezuela ocupando el cuarto lugar y por ultimo Paraguay relegado a la última posición ocupando el lugar 95 de 177 naciones consideradas. (ver Cuadro No. 2).

Cuadro No. 2. Asimetrías en desarrollo

\begin{tabular}{|c|c|c|c|}
\hline Países & Índice de Desarrollo Humano (IDH) 2005 & IDH (Ranking Mundial) 20055 & $\begin{array}{c}\text { Competitividad Ranking } \\
\text { Mundial, 2005-2006 }\end{array}$ \\
\hline Argentina & 0.869 & 38 & 54 \\
\hline Uruguay & 0.852 & 46 & 70 \\
\hline Brasil & 0.800 & 70 & 57 \\
\hline Venezuela & 0.792 & 74 & 84 \\
\hline Paraguay & 0.755 & 95 & 102 \\
\hline
\end{tabular}

Fuente: Elaboración propia con datos del Informe sobre Desarrollo Humano (2005); y The Latin America Competitiveness Review (2006). 
Del mismo modo, Argentina sigue siendo el país más competitivo seguido ahora de Brasil ocupando las posiciones 54 y 57 respectivamente, Uruguay se sitúa en la posición 70, luego Venezuela en la posición 84 y por ultimo Paraguay queda relegado nuevamente a la posición 102. Según se ha observado en los cuadros anteriores Argentina y Brasil son las grandes potencias económicas del MERCOSUR, Uruguay y Paraguay serían los dos países más débiles y por su parte Venezuela quedaría en un punto intermedio entre los "países grandes" y los "países pequeños". Por ello, no hay duda que en América del Sur el PIB de Argentina y Brasil tiene una gran brecha asimétrica con respecto a los otros países. (ver Gráfico No. 1)

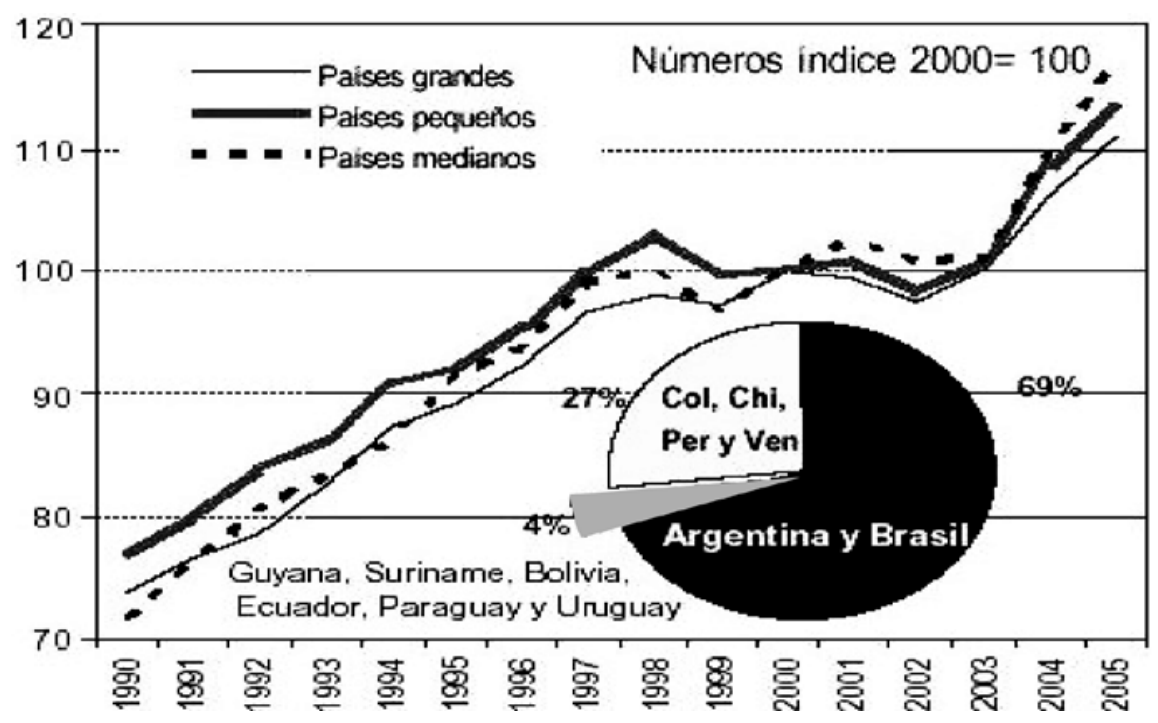

Gráfico No. 1. Evolución del Producto Interno Bruto, 1990-2005

Fuente: CEPAL (2006).

El gráfico No. 1 muestra la gran diferencia asimétrica del PIB que existe en América del Sur en donde sólo Argentina y Brasil poseen el 69\% del PIB de la región, Colombia, Chile, Perú y Venezuela solamente tienen el 27\% y los 6 países más pequeños restantes (Guyana, Suriname, Bolivia, Ecuador, Paraguay y Uruguay) sólo alcanzan un 4\%.

Por tal razón, es indudable que en el MERCOSUR las dos grandes potencias económicas son Argentina y Brasil, las cuales poseen grandes asimetrías con las economías de Uruguay y Paraguay que poseen un PIB con muy poco peso en dicho esquema de integración.

En este mismo sentido, cuando se compara el desempeño en el primer quinquenio de la presente década con el registrado en el primero de los noventa, el crecimiento económico es inferior en forma generalizada (ver Cuadro No. 3). 


\section{CIENCIAS SOCIALES}

Cuadro No. 3. Asimetrías en cuanto a desempeño económico, producción, desempleo y evolución del índice de precios al consumidor, 1991-1995, 1996-2000 y 2001-2005

(Expresado en porcentajes)

\begin{tabular}{|c|c|c|c|c|c|c|c|c|c|}
\hline \multirow{2}{*}{$\begin{array}{c}\text { Indicadores } \\
\text { Países }\end{array}$} & \multicolumn{3}{|c|}{\begin{tabular}{c} 
Crecimiento del PIB \\
en moneda Constante de \\
\cline { 2 - 11 }
\end{tabular}} & $\mathbf{1 0 0 0}$ & \multicolumn{3}{c|}{$\begin{array}{c}\text { Desempleo } \\
\text { En porcentajes de la PEA }\end{array}$} & \multicolumn{3}{c|}{$\begin{array}{c}\text { Inflación } \\
\text { Tasa anuales medias }\end{array}$} \\
\cline { 2 - 12 } & $\mathbf{1 9 9 1 - 1 9 9 5}$ & $\mathbf{1 9 9 6 - 2 0 0 0}$ & $\mathbf{2 0 0 1 - 2 0 0 5}$ & $\mathbf{1 9 9 1 - 1 9 9 5}$ & $\mathbf{1 9 9 6 - 2 0 0 0}$ & $\mathbf{2 0 0 1 - 2 0 0 5}$ & $\mathbf{1 9 9 1 - 1 9 9 5}$ & $\mathbf{1 9 9 6 - 2 0 0 0}$ & $\mathbf{2 0 0 1 - 2 0 0 5}$ \\
\hline MERCOSUR & $\mathbf{4 , 8}$ & $\mathbf{6 , 9}$ & $\mathbf{2 , 8}$ & $\mathbf{6 , 1}$ & $\mathbf{8 , 2}$ & $\mathbf{1 0 , 6}$ & $\mathbf{6 5 8 , 7}$ & $\mathbf{4 , 3}$ & $\mathbf{9 , 6}$ \\
\hline Argentina & 7,1 & 7,9 & 3,5 & 10,4 & 14,9 & 15,9 & 22,9 & $-0,3$ & 12,3 \\
\hline Brasil & 3,8 & 6,5 & 2,5 & 5,1 & 6,7 & 9,4 & 1002,0 & 6,3 & 8,6 \\
\hline Paraguay & 4,8 & 3,9 & 2,7 & 5,0 & 8,3 & 11,3 & 15,8 & 8,6 & 9,0 \\
\hline Uruguay & 4,9 & 6,8 & 2,0 & 9,1 & 11,7 & 14,9 & 54,6 & 11,5 & 10,4 \\
\hline Venezuela & 4,3 & 2,9 & 2,0 & 8,6 & 12,7 & 14,9 & 47,3 & 40,8 & 20,8 \\
\hline
\end{tabular}

Fuente: CEPAL, (2006).

Tal como se ha visto, en relación con la tasa el desempleo en base a la Población Económicamente Activa, cabe resaltar el hecho de que en el período 2001-2005, todos los países tuvieron tasas elevadas, que hasta superaban el promedio a excepción de Brasil que posee una tasa de desempleo de un solo digito. Argentina es el que posee la mayor tasa de desempleo, seguido de Venezuela y Uruguay con el mismo valor, en este caso Paraguay es el segundo país con la tasa de desempleo más baja.

Por otro lado, en relación con la tasa de inflación se puede observar, que en el periodo 2001-2005 la tasa de inflación de Venezuela es la más alta con un 20,8\%, casi duplicando a Argentina el cual es el segundo país con más alta tasa de inflación, Uruguay por su parte posee el 10,4\%, Paraguay 9\% y por último Brasil con la menor tasa de inflación del MERCOSUR con un $8,6 \%$. Se puede notar igualmente si se compara la tasa de Venezuela que es la más alta con la tasa de Brasil que representa la más baja, que la tasa venezolana es casi dos veces y medio la tasa brasilera.

Así pues, a la luz de estos indicadores, los autores consideran que el MERCOSUR es una región con crecimiento si se quiere insuficiente y volátil, que dista mucho de lograr alcanzar trayectorias de convergencia hacia los patrones de crecimiento de los países desarrollados. Esta situación aumenta por la creciente heterogeneidad productiva, ya que los países más pequeños tienen mayor dotación de factores en productos agrícolas: Uruguay y Paraguay (ver Cuadro No. 4). 
Cuadro No. 4. Desglose del PIB por grandes sectores económicos

(Expresado en porcentajes del PIB)

\begin{tabular}{|l|l|l|l|l|l|}
\hline \multicolumn{1}{|c|}{ Países } & \multicolumn{1}{c|}{ Agricultura } & \multicolumn{1}{c|}{ Minería } & \multicolumn{1}{c|}{ Industria } & \multicolumn{1}{c|}{ Servicios } & \multicolumn{1}{c|}{ Total } \\
\hline Brasil & 9,3 & 3,8 & 32,6 & 54,3 & 100,0 \\
\hline Argentina & 9,9 & 5,4 & 32,1 & 52,7 & 100,0 \\
\hline Uruguay & 11,4 & 0,2 & 24,1 & 64,2 & 100,0 \\
\hline Paraguay & 22,4 & 0,1 & 21,9 & 55,6 & 100,0 \\
\hline Venezuela & 3,6 & 19,4 & 38,7 & 38,3 & 100,0 \\
\hline
\end{tabular}

Fuente: CEPAL, (2006).

Como se puede observar, en Venezuela la mayor cantidad del PIB se concentra en el sector de industria y de servicios con el 38,7\% y 38,3\% respectivamente, luego el sector minero con un 19,4\% y por último el sector de agricultura con sólo el 3,6\%. Por su parte, Argentina, Brasil, Uruguay y Paraguay concentran su PIB principalmente en el sector de servicios; sin embargo Paraguay concentra un importante porcentaje del PIB en el sector de agricultura. De igual manera se puede observar que el sector fuerte del PIB de Venezuela respecto a los países del MERCOSUR es el sector de minería, frente al más cercano (Argentina) que sólo concentra el 5,4\%, seguido de Brasil con 3,8\%, Uruguay 0,2\% y Paraguay 0,1\%.

\section{Criterios sobre la Balanza Comercial de Venezuela con el MERCOSUR}

El estudio de la Balanza Comercial se constituye uno de los principales indicadores macroeconómicos que deben analizar con suma cautela todo los países a la hora de establecer las prácticas habidas en el Comercio Internacional, de manera que al darse desequilibrios en estos indicadores, es decir, en las importaciones y exportaciones estos generarían como consecuencias efectos comprometedores en otra variables macroeconómicos manifestando distorsiones importantes en la economía.

En este sentido, se presenta los cuadros 5 y 6 de la Balanza Comercial de Venezuela con MERCOSUR durante el período 2000 - 2009 donde se puede aprecia que el intercambio comercial ha sido altamente desfavorable para Venezuela, debido al creciente consumo de bienes originarios de países, en este esquema particularmente los elaborados en Brasil y Argentina, quienes también se vieron altamente beneficiados a partir de la firma del ACE N 59 y la consecuente salida de Venezuela de la CAN conjuntamente con la firma del Protocolo de Adhesión de Venezuela al MERCOSUR, implica la generación de mecanismos que impulsaron de manera significativa la colocación de muchas exportaciones de bienes de los países integrantes del bloque sudamericano al territorio venezolano, quienes ya desde el año 2004 representaban más del 9o\% de la participación del intercambio comercial entre estas partes y tal tendencia ha seguido en aumento desde entonces, reflejándose un 97\% de participación de intercambio comercial que proviene de los países MERCOSUR, frente a solo un 3\% de exportaciones venezolanas dirigidas hacia ese mercado. 
Cuadro No. 5. Importaciones / Exportaciones Venezuela - MERCOSUR (Millones US\$) $2000-2009$

\begin{tabular}{|c|c|c|c|c|c|c|c|c|c|c|}
\hline $\begin{array}{c}\text { VENEZUELA - } \\
\text { MERCOSUR }\end{array}$ & 2000 & 2001 & 2002 & 2003 & 2004 & 2005 & 2006 & 2007 & 2008 & 2009 \\
\hline Importaciones & 1029,3 & 1276,6 & 980,8 & 780,0 & 1658,3 & 2480,0 & 3780,2 & 5664,0 & 5538,9 & 4334,7 \\
\hline Exportaciones & 178,2 & 228,3 & 178,4 & 151,0 & 187,6 & 214,8 & 186,1 & 161,9 & 147,5 & 118,2 \\
\hline Total & 1207,5 & 1504,9 & 1159,2 & 931,0 & 1845,9 & 2694,8 & 3966,3 & 5825,9 & 5686,4 & 4452,9 \\
\hline
\end{tabular}

FUENTE: INE, Cálculos propios.

Cuadro No. 6. Proporción Porcentual Importaciones / Exportaciones Venezuela - MERCOSUR (Millones US\$) $2000-2009$

\begin{tabular}{|c|c|c|c|c|c|c|c|c|c|c|}
\hline $\begin{array}{c}\text { VENEZUELA - } \\
\text { MERCOSUR }\end{array}$ & $\mathbf{2 0 0 0}$ & $\mathbf{2 0 0 1}$ & $\mathbf{2 0 0 2}$ & $\mathbf{2 0 0 3}$ & $\mathbf{2 0 0 4}$ & $\mathbf{2 0 0 5}$ & $\mathbf{2 0 0 6}$ & $\mathbf{2 0 0 7}$ & $\mathbf{2 0 0 8}$ & $\mathbf{2 0 0 9}$ \\
\hline Importaciones & $85 \%$ & $85 \%$ & $85 \%$ & $84 \%$ & $90 \%$ & $92 \%$ & $95 \%$ & $97 \%$ & $97 \%$ & $97 \%$ \\
\hline Exportaciones & $15 \%$ & $15 \%$ & $15 \%$ & $16 \%$ & $10 \%$ & $8 \%$ & $5 \%$ & $3 \%$ & $3 \%$ & $3 \%$ \\
\hline
\end{tabular}

FUENTE: INE, Cálculos propios

\section{Conclusiones}

La República Bolivariana de Venezuela, toda vez que ingrese al MERCOSUR, tendrá que modificar su política comercial y acervo normativo para ajustarla a los requerimientos contemplados por el bloque regional suramericano para la adhesión de potenciales miembros. MERCOSUR aún no ha aprobado el ingreso de La República Bolivariana de Venezuela, solo el Parlamento de Paraguay no se ha pronunciado para tales efectos y tiene en sus manos decidir si Venezuela definitivamente integrará dicho esquema. Este proceso de ingreso debe ser estudiado con mucha cautela, ya que se espera solucionar aspectos en materia arancelaria, comerciales y económicos.

La República Bolivariana de Venezuela debe adoptar la Nomenclatura Común del MERCOSUR (NCM) y el correspondiente Arancel Externo Común (AEC) a más tardar cuatro años después de haber sido aprobado el ingreso por la totalidad de los miembros del bloque (MERCOSUR).

El Protocolo de Adhesión una vez entre en vigencia, Venezuela dispondrá de un lapso no mayor de cuatros años con los países del bloque para lograr internalizar a su marco normativo una serie de compromisos y activar los mecanismos del libre comercio intraregional. Estos lapsos son insuficiente si se analiza que las acciones que reviste reformar la política comercial y adecuarlas a las exigencias del MERCOSUR es un tanto complejo que debe estudiarse y cumplir con una serie de procesos que conlleve a una correcta armonización de actividades y normas, dirigidas a proteger el sector productivo nacional y de esta manera minimizar los posibles impactos que podrían originar el ingreso a este bloque. 
Venezuela al formar parte de este bloque, sin lugar a dudas su balanza comercial generará una dinámica relevante, es decir las importaciones y las exportaciones se incrementarán, la población podrá consumir más bienes y servicios los cuales se encontrarán con menos grado de protección, de manera que las empresas adecuarán su producción a la competencia externa de los socios de MERCOSUR, lo que podría originar una caída parcial de la producción ineficiente. Así pues el aumento de los niveles de consumo, la disminución de la producción ineficiente y el crecimiento de las importaciones en el país sería el efecto de creación de comercio si Venezuela participa activamente en el MERCOSUR.

No obstante, dicha creación de comercio podría venir acompañada con una desviación de la misma, lo que ocasionaría que productos de mayor calidad que existían en el mercado nacional ahora se verán desplazado por productos importados directos del bloque.

En otro orden de ideas, las asimetrías dadas para con el bloque y la República Bolivariana de Venezuela son muy notorias, Venezuela tiene un potencial que en materia energética y minera que la caracteriza. Si se observan los indicadores se puede apreciar que Venezuela tiene aun muchas debilidades en cuanto a desarrollo humano se refiere, nivel de competitividad, Producto Interno Bruto (PIB), inflación y niveles de pobreza.

Es importante destacar que los países que conforman el MERCOSUR y Venezuela, han presentado desigualdades estructurales y políticas muy significativas, en cuanto a Brasil, es un país muy competitivo y con un índice de desarrollo alto, a pesar de ser un país con uno niveles de pobreza relevante; Paraguay, es el país más pobre, el segundo en poseer menos tasa de inflación y una de las tasas de desempleo más baja y además se encuentra muy por encima de Uruguay en cuanto a la producción de Energía.

Las asimetrías en el MERCOSUR han sido asfixiantes para los miembro del bloque, empero los mecanismos para paliar un poco dichas desigualdades, tal es el caso del FOCEM, este no ha aportado mayores beneficios para sus integrantes.

Es importante destacar que la creación del FOCEM representa un paso sumamente alentador para con el tratamientos de las asimetrías debido a que se ha hecho un reconocimiento oficial de la existencia de estas desigualdades que reclaman la constitución de políticas que contrarresten por parte de los organismos competentes, para que de esta forma exista una mayor igualdad entre los países miembro del bloque y de esta manera poder promover el desarrollo de los países con menor grado de crecimiento y desarrollo, logrando así la profundización de dicho esquema integracionista que conlleve al desarrollo de los países. 


\section{Lista de referencias}

Vásquez, C, (2002). Implicaciones para el comercio marítimo venezolano de constituirse en Zona de Libre Comercio con MERCOSUR, Trabajo final de grado, Dirección de investigación y Postgrado de la Universidad Marítima del Caribe. Caracas

Terra, M. (2008). Asimetrías en el Mercosur: ¿Impedimento para el crecimiento?. Documento en línea. Disponible: http://www.redmercosur.org.uy. Consulta 2009, Abril 15

Constitución de la República Bolivariana de Venezuela (2009). Gaceta Oficial de la República Bolivariana de Venezuela, $N^{\circ} 5.453$ (Extraordinario), Marzo 24 del 2000

Instituto Nacional de Estadística. Documento en línea. Disponible en http://www. ine.gob.ve,

Heredero, M. (2001). La Organización Mundial del Comercio Frente al acuerdo General sobre Aranceles y Comercio. Documento en línea. Disponible: http://www.revistasice.com Consulta: 2009, Abril 16.

Protocolo de Adhesión de la República Bolivariana de Venezuela al MERCOSUR. (2006). Documento en línea. Disponible: http://www.mercosur.int/msweb/ Portal\%20Intermediarios/ES/index.htm. Consulta 2009, Mayo 15.

Tratado de Asunción, (1991). Documento en línea. Disponible: http://www.mercosur. Int/msweb/Portal\%2oIntermediarios/ES/index.htm. Consulta: 2009, Abril 26. 